\title{
SALUD-EDUCACIÓN: LO QUE PUDO SER Y NO ES
}

Jesús Edilberto Rodríguez Rosero * Maria das Graças Carvalho Ferriani **

ROSERO, J.E.R.; FERRIANI, M.G.C. Salud-Educacion: Lo que pudo ser y no es. Rev.latino-am.enfermagem, Ribeirão Preto, v. 5, p. 57-62, julho 1997.

Partiendo de su conocimiento y trayectoria experiencial, el autor hace una crítica a la concepción y cosmovisón práctica de la medicina, la salud, la educación y el papel ingénuo de los ciudadanos. Con argumentaciones sintéticas intenta despertar interrogantes y dar una interpretación a las casuas de la ineficacia del sistema de salud y su severo dislocamiento de lo social frente al avance científico y tecnológico.

\section{UNITERMOS: crítica, salud, educación}

A lo largo de los 22 años de experiencia laboral y profesional en diversos campos de la educación y de la salud en los niveles existentes, o sea Hospitales Universitarios, Servicios de Salud, capacitación de Promotores de Salud, de Auxiliares de Enfermería, de profesionales de Enfermería en el Nivel de Pregrado y Postgrado, en la Coordinación de Centros y Puestos de Salud, en lo que se refiere a los Programas que se ofrecen a la Comunidad, paulatinamente he encontrado innumerables situaciones que generan múltiples inquietudes. Esas inquietudes se reflejan en las siguientes preguntas: En qué grado, la consciencia determina las condiciones materiales de la existencia, específicamente la salud?

Porqué las personas en su vida cotidiana actúan inconsistentemente, con respecto a los principios elementales útiles para cuidar la salud?

Puede una persona culta en salud, preservarla, si vive en un tugurio?

Como ser inteligente, cuál es la responsabilidad que tiene una madre, frente a su propio cuidado y al de su núcleo familiar, principalmente frente a sus hijos en sus primeros años?

Es innegable que la salud es un bien deseable por todos y con más intensidad por la familia con respecto a sus seres queridos, es decir la colectividad desea vivir sin enfermar, sin embargo a través de la historia siempre se intentó dar la salud, teniendo como punto de referencia la institución de salud, a su vez en el último siglo, se ha pensado en que para proponer algo bueno o eliminar algún mal, es necesaria la educación, al parecer usando como estrategia la educación se puede hacer todo.

La dura realidad que se conoce, tangible en los hospitales públicos, es que la mayoría de enfermedades infantiles son evitables o prevenibles, unas con acciones gubernamentales como la optimización de los servicios públicos, otras con alimentos de buena calidad, otras son prevenibles con un poco de educación, la mayoría con acciones acertadas por parte de quien cuida los niños, aún en precarias condiciones ambientales. No obstante toda la inversión en salud y la educación que se ha ejecutado no han logrado cambios comportamentales que se reflejen en la disminución significativa de las morbilidades.

Los sociosalubristas afirman, que las condiciones sociales históricamente determinadas, la privación al acceso a los bienes materiales como la comida y a los bienes culturales como la educación generan las condiciones para que la gente enferme. En los discursos de muchos políticos y médicos aun se escucha decir: Es que faltan centros, puestos de salud, hospitales, médicos, y recursos para atender a los enfermos. Tengo la convicción de que las soluciones no están en en la creación de hospitales Materno Infantiles o Universitarios.

En muchísimas visitas que he efectuado en

\footnotetext{
* Profesor Titular Universidad de Los Llanos-Colombia. Lic. Enf. U. Nal. de Colombia.M/Sc. Desarrollo Educativo y Social. UPN-CINDE. Bogotá. Estudiante de Doctorado de la Escuela de Enfermería de Ribeirão Preto de la Universidad de São Paulo. Brasil

** Orientadora - Professor Associado do Departamento de Enfermagem Materno-Infantil e Saúde Pública de la Escuela de Enfermería de Ribeirão Preto de la Universidad de São Paulo. Brasil
} 
Colombia a sectores poblacionales aislados y pobres, en los departamentos de Cundinamarca, Chocó, Guaviare, Santander, Meta y Nariño, en donde después de todos los saludos rituales de parte y parte, de manera directa o indirecta se llega a la siguiente pregunta: Bueno y cómo están en salud? La respuesta es unánime: "Ah no, en eso estamos muy mal; aquí no tenemos médico, ni siquiera un promotor o enfermera, menos un puesto de salud.

A juzgar por la anterior, si de dar solución en salud se trata; es aquí donde comienza lo complicado, porque con esta visión medicalizada impuesta por el poder de la medicina concuerdan las comunidades.

Se olvidó que la salud es un bien deseado por todos y en consecuencia las personas deben tener la disponibilidad para luchar por la construcción de su propia salud y la de sus seres queridos y el estado la obligación de proveer las condiciones necesarias para lograrla.

La realidad práctica indica que la educación en salud y para la salud que implica un encuentro cultural permanente con revalorización de la persona y su comportamiento como responsable en parte de su própia asistencia, no fue tenido en cuenta, la educación informativa o impositiva, no alcanza la generación de actitudes y prácticas que favorezcan el mejoramiento de la salud. La concepción simplista de la educación, hizo que se olvidara que lo más complejo sucede cuando tiene que llevarse el concepto de educación a su operacionalización, usando para ello técnicas, métodos, ayudas, aptitudes y actidudes individuales, todos bajo un enfoque pedagógico conciso, un momento adecuado y con permanente consistencia interna.

Además la mistificación del médico por parte de los medios de comunicación y la sociedad ha logrado que las comunidades crean que la salud es: Puesto de salud, médico y medicamentos; muchos de los profesionales de la salud también creen que el núcleo salvador de la salud colectiva se encuentra en los programas que ofrece el sistema de salud, medicalizado y con énfasis casi absoluto en patología, sintomatología y tratamiento, tal como se conoce.

Este concepto distorsionado, no se generó espontáneamente, sino por el ejercicio del poder de dominio intelectual y económico por parte de los dueños de la medicina y de las fabulosas industrias de la enfermedad. La mayoría de los médicos acreditan o al menos actúan, basados en la medicina curativa, asistencial, individual y rentable, creen que usando la tecnología como forma de responder a la enfermedad, la ciencia tendrá condiciones de vencer la enfermedad y la muerte, valiendose del estado que suministra dinero y poder; insidiosamente la sociedad que hace de ellos los más prestigiados y lucradores del sistema, no han conseguido respuestas a los problemas de la salud colectiva, por el contrario en su mayoría luchan por una implantación de la medicina privada con otras formas sutiles o disimuladas de acrecentar su riqueza.

Es cierto que la situación socioeconómica influye en forma importante en el estado de salud de la población, pero esta situación solo es entendible a la luz de la sobrexplotación y el marginamiento, aunados a la injusta repartición de las riquezas nacionales y la explotación de los países más fuertes, que han dado origen a la formación de los grandes cinturones de miseria en donde las condiciones ambientales son insalubres, coexistiendo a escasos metros con fabulosas mansiones, edificios y todo tipo de ostentación de riqueza y comodidad.

Cualquier reflexión sobre la salud y la enfermedad, lleva a conclusiones de que se ha vivido en permanente abandono personal, estatal y de la comunidad, en contraposición a los grandes adelantos de la ciencia y la gran inversión en salud curativa, con el esquema "si te quejas te doy salud".

Por otro lado se desconoce que los cambios en los comportamientos negativos precisan de una introyección del problema y para que esto ocurra, es base indispensable que exista la posibilidad de que la situación de riesgo sea detectada y valorada por la población, además de una voluntad positiva hacia el cambio. Estos comportamientos negativos e inconsistentes con respecto al conocimiento científico, que conducen a perder la salud, no pueden quedarse enmarcados en explicaciones de subdesarrollo, ignorancia, marginamiento, carencia de educación formal, torpeza o falta de voluntad de los protagonistas; pues son en gran medida la consecuencia de una raigambre cultural, de la concepción misma del desarrollo y el enfoque de la medicina, así como de la mínima ejecución de una educación sanitaria ecológica, cuya interacción total se materializa en los diferentes comportamientos de los individuos.

Recordemos también que todo el mundo construido e internalizado por los individuos es estractado de la tradición y de la macrobservación empírica; de tal manera que los comportamientos ilógicos a la luz de la racionalidad y lógica de los profesionales de la salud, son plenamente lógicos y racionales bajo la perspectiva conceptual, perceptual, social e histórica de los protagonistas.

El logro de la quiebra de esa falsa conciencia; necesita, de igual forma, la troca de paradigmas y estereotipos de la medicina ortodoxa, por ejemplo es necesario pasar de procesos cortos y localizados de educación en salud a procesos masivos, permanentes y sistemáticos, abandonando el enfoque curativo clínico asistencial, reemplazándolo por otro autoconstructivo comunitario preventivo, ecológico; cambiar el concepto de que la medicina es ante todo, porque los hombres se sienten enfermos, por, la 
medicina es ante todo porque el hombre necesita prevenir la enfermedad, construir la salud y por ende su felicidad.

Si aceptamos el proceso salud-enfermedad es una determinación social-histórica, que consiste en un desequilibrio físico y/o psicológico, resultante de las condiciones de alimentación, habitación, educación, renta, recreación, acceso al agua potable, eliminación adecuada de excretas y basuras, fácil accceso a los servicios de salud, en mejoramiento genético, estamos siendo parciales en la concepción; supongamos por ejemplo que todas las condiciones fisicas son optimas, esto no garantiza un optimo estado de salud, porque creo que el comportamiento individual-colectivo, es una determinante con un índice significativo, que debemos incorporar en discurso de la salud colectiva.

Así es posible retratar una visión totalizante, que la hace diferente por el hecho de considerar a la persona autoconstructora y factor determinante olvidado por los pensadores de la salud pública, he aquí que existe en realidad un control posible, sobre el propio bienestar, constituyen este autocontrol las conductas o comportamientos, a manera de una autodefensa de la própia población, VALLA $(1986)^{8}$, afirman que en las condiciones de la vida en las favelas, se vive en estado de "Emergencia Permanente".

El comportamiento de los individuos en estado de emergencia permanente, si es basado en el conocimiento y la racionalidad, necesariamente es digno de ser reconocido y dimensionado, en forma contraria si el predominio es un comportamiento errado, necesita ser dimensionado para elevarlo a nivel de la conciencia revalorado y en lo posible modificado u omitido.

Cuando se habla de comunidad debe entenderse que se trata de un grupo numeroso de personas que habitan un área geográfica determinada, por lo tanto comparten parte de su historia, costumbres, ambiente, carencias, necesidades, problemas; de igual forma padecen en grandes proporciones de las mismas enfermedades, al mismo saneamiento básico, están expuestos a los mismos riesgos, al mismo desgaste, al mismo trato, e incluso a las mismas opciones de alimentación y atención en salud.

En condiciones de pobreza se presentan muchas limitaciones, que a su vez generan situaciones interrelacionadas ineludibles, el desarrollo forzoso de la cotidianidad exige respuestas inmediatas y obligatorias, para cuidar o abandonar su cuerpo y su entorno, la respuesta dada es necesariamente fruto de factores personales e impersonales de la concepción del fenómeno y de la percepción que se tiene de él.

En el área de la salud se presentan numerosos comportamientos legados de la tradición oral que en gran proporción son erróneos, otros son producto de principios derivados de la macrobservación, la mayoría son omisiones, por la escasa percepción o la valoración inadecuada del estado de emergencia permanente, esto facilita la permanencia de enfermedades y su propagación.

El concepto de comportamiento débese entender desde una perspectiva holística, es decir es la capacidad de dar existencia al mundo exterior, con la integración de toda la información perceptiva, recibida por los sentidos en forma directa o indirecta, mediata o a largo plazo.

El encuentro educativo en salud se constituye en un encuentro cultural por excelencia, que si quiere ser efectivo, exige una permanencia en las acciones y una insistencia que logre un sentimiento de pertenencia, para que no se convierta en una mera forma de imposición y de poder, pues la democratización del conocimiento técnico implica una revalorización permanente que transforme acciones negativas en positivas para el mejoramiento de la salud, es decir el usuario necesita ayudar a construir la salud, necesita involucrarse en la acción con un concepto de desarrollo humano construido desde lo educativo.

Es claro que el agente educativo debe poseer capacitación técnica y pedagógica, para que además de permitir, facilite que el usuario asuma el papel que de este contenido se desprende.

Al respecto en reciente investigación de la Doctora BENSEN (1995) ${ }^{1}$ encontró lo siguiente:

“A partir da implantação do SUS, uma nova concepção de saúde vem sendo socialmente construída, onde a dimensão educativa do trabalho do enfermeiro está mais presente e faz emergir um novo perfil do enfermeiro com um forte compromisso sócio-político, devendose além da assistência, trabalhar também a consciência do usuário"... "Verificou-se que o enfermeiro, enquanto trabalhador, mesmo enfrentando as adversidades do cotidiano de sua prática e o poder institucional vem aprendendo e se construindo em e pelo seu trabalho..." “O enfermeiro precisa estar alerta para começar a perceber as situações concretas que se apresentam no seu cotidiano. A dimensão pedagógica do trabalho, muitas vezes não é percebida em forma clara pelos enfermeiros, numa perspectiva de transformação, sendo este um aspecto limitador de sua prática." "Um novo papel do enfermeiro de cunho mais educativo, deve ser entendido como um buscar constante de forma mais participativa com os usuários do serviço de saúde, levando-os a uma melhor visão acerca de seu processo de viver, passando sua prática a ser visualizada como um embrião de transformação”. 
La anterior investigación reivindica y explica desde su perspectiva, el principio propio de el profesional de enfermería, y le imprime la dimensión pedagógica al trabajo del profesional de enfermería, con una perspectiva de transformación y algo más profundo aún, trabajar sobre la consciencia del usuario; que bello compromiso. Esto exige en la formación del profesional un contenido profundo de una pedagogía emancipadora y el desarrollo de habilidades comunicativas de alta competencia.

VALLA (1986)" , afirma: "Diante da situção de emergência permanente..., os serviços de saúde e demais serviços básicos devem transformar-se em sistemas locais de defesa-promoção coletiva da vida. Tal mudança pressupõe, contudo uma nova relação entre os profissionais que atuam nesse nível”. En el mismo sentido MELLO (1993) ${ }^{4}$ afirma:

\begin{abstract}
"Seria importante o serviço valorizar a figura da mãe no processo de cuidar das crianças, enquanto aquela que efetivamente presta os cuidados gerais à criança no dia-a-dia, reconhecendo alguns sinais e sintomas de certas doenças, acompanhando o crescimento, fornecendo alimento, amor e educação aos filhos. Os profissionais de saúde necessitam repensar a relação usuário/serviço, no sentido de reconhecer as percepções trazidas pela população e se envolverem com a realidade de saúde e de vida da população ...”
\end{abstract}

Desde este interés emancipatorio de transformación permanente, que presupone un nueva relación, exige cuantificar, cualificar y revalorar otros parámetros de la realidad percibida por las comunidades, para la planeación de acciones educativas.

Es bien aceptado que los cambios comportamentales son conservados, cuando las personas creen, que son hechos a partir de su própia disposición interior y hace sentido para ellas y siempre será olvidado y rechazado lo que es el producto de imposiciones o manipulaciones hechas desde el exterior.

Sin embargo no es suficiente la apropiación de contenidos, si en un momento superior y posterior los individuos no hacen una readaptación y recreación de lo apropiado a otras situaciones particulares, es necesaria una conciencia transitiva crítica, en donde se está dispuesto a revisiones y reinterpretaciones, orientando la actividad humana hacia fines deseados, caracterizandose la conciencia crítica como el estado en que se es capaz de investigarse a sí mismo, con una tendencia a problematizar y a discutir todo aquello que parece obvio y verdadero, que no solamente acepta el cambio, sino que está dispuesto a cambiar.

En el aspecto pedagógico y comunicativo, es necesario reconocer que es lo más débil, por parte de los profesionales de la salud, pues los nutricionistas, fisioterapeutas, odontólogos, médicos, reciben su grado y de inmediato se convierten en educadores de salud y en otros casos profesores; en lo pertinente de los profesionales de enfermería esta falencia ha sido corregida en los últimos años.

Por desconocimiento y como herederos de un conductismo autoritario, quienes ejercemos la intención de enseñar, actuamos como absolutos poseedores del saber, con fuerte tendencia a repetir la actitud de nuestros profesores y ejercer el poder de que nos inviste el ser vistos con el halo de médico sanador y salvador de todos los males y dolores presentes, predeterminando absolutamente todos los contenidos y códigos lingüisticos que creemos necesario que "ellos" aprendan; para que mis lectores profundicen mejor a continuación estracto algunos conceptos del gran pensador BERSTEIN $(1971)^{2}$.

"El código elaborado se caracteriza por ser adecuado a un referente específico; facilitador de la construcción y de intercambio de símbolos individuales, productor de un nivel más alto de organización estructural y léxica; poseedor de las capacidades de transmisión de significados explícitos y de elaboración más fácil de experiencias individuales a través de transmisión de reglas y principios. El código restricto, en contrapartida, es definido por el énfasis dado en la inmediatización de la relación, por reducción del planeamiento verbal, por la transmisión de reglas y no de principios, por el uso de expresiones faciales como los principales indicadores de cambios de significados, por su tendencia a la impersonalidad, por la falta de adecuación a un referente específico, por que su contenido tiende a ser concreto, descriptivo y narrativo".

Cada lector que posiblemente ha ejercido como profesor u orientador, con variedad de públicos, reflexionará sobre su propia actitud, tenemos que aceptar que en el área de la salud debe darse primacía a la acción comunicativa; pero por el contrario este encuentro se hace siempre con el habla mandativa de reglas específicas, sin referente a la cosmovisión del paciente, con códigos estrictos e inmediatistas, que necesariamente conducen al fracaso en el objetivo de lograr que haya un aprendizaje sobre los cuidados o medidas preventivas tan conocidas en el ambiente académico de la salud pública para ser reelaborado y mejorar la conexión entre teoría y mundo de la vida, es decir ser aplicado en la vida cotidiana. Se olvida también que la verdadera inserción cultural exige estar cerca de la gente, de sus necesidades, de sus motivaciones, 
de sus percepciones de su lenguaje, de su propia lectura del mundo cotidiano, y en interacción dialógica, lo que implica la problematización del propio conocimiento, el empírico y el científico; la indiscutible relación del individuo con su realidad concreta basada en la macrobservación, en la cual se genera el conocimiento y sobre cuya realidad incide, para lograr así comprenderla, explicarla y acaso transformarla. Esto, cuando la autoridad suprema del acto médico, al menos intenta comunicación.

He hablado de la educación y la comunicación por ser ellos, claves en la tarea de construcción de la salud, en todos los momentos de la vida; en lo que se refiere al periodo de socialización secundaria y por ser allí donde podemos actuar, pero los espacios más influyentes e importantes para esa tarea, están ocupados por otros agentes en el jardín infantil, la escuela primaria y el hogar, actualmente poseedor de receptores de comunicación masiva que manipulan, borran y crean valores o antivalores. Para REY (1987) “' "La socialización es aquel proceso mediante el cual todo miembro de determinado grupo social, se hace partícipe de sus valores, creencias, sistemas de percepción, sistemas normativos, formas de control social", el mismo autor (1991) ${ }^{7}$ afirma que: "La interacción de aprendizaje se ejecuta en el espacio real e inmediato de la vida cotidiana; es allí donde se experimenta el poder, la ideología, la alienación, la transformación y la posibilidad de cambio".

Toda la socialización primaria ocurre en los primeros 7 años de vida, en esta variable tan grande y definitiva, que se hace en espacio real y cotidiano del hogar y es aquí donde se adquieren los valores, creencias, percepción; es decir aquí están las plantillas para el comportamiento posterior, incluso de la estética, el amor, el respeto al otro, la valoración y respeto de la naturaleza, etc.

Con respecto al problema de eficiencia de los programas educativos que intentan en forma masiva llegar al seno de las comunidades, existe en Colombia el programa SUPERVIVIR, realizado por los profesores y estudiantes de $9^{\circ}$ y $10^{\circ}$ grado escolar, al estudiar lo que sucede con ese programa; GUEVARA \& RODRIGUEZ $(1993)^{3}$. Afirman lo siguiente:

"El bajo nivel de apropiación teórica lograda, conduce a un diálogo intersubjetivo escaso y restringido, en consecuencia no puede crear una conciencia colectiva, sobre el sentido de un aprendizaje para la vida; no puede haber una reelaboración de conceptos... Ante una apropiación teórica deficiente, es de esperar una aplicación práctica también deficiente, lo que se confirmó en la observación de 100 familias beneficiarias, donde el $57 \%$ de las conductas observadas fueron calificadas como regulares $o$ malas, es decir el aporte familiar para la construcción de la salud es altamente deficiente; este resultado no es dependiente solo de la acción educativa del vigía, sino de una multicausalidad que algunos estudiosos denominan patología social. Este distanciamiento entre la verdad teórica aprendida y las conductas negativas incoherentes en salud, en una alta proporción obedecen a la introyección de una estructura cultural colectiva, carente de principios para construir la salud... El paradigma de enseñar para transformar, es solo un estereotipo de verdad, sería mejor y más real enseñar a reflexionar, para conocer y transformar; el nivel cognitivo logrado ascendería hasta develar el accionar incongruente, y aterrizaría al individuo en otras verdades y otras conductas, la estrategia metodológica debería dirigirse a auxiliar conciencias, para que ellas puedan reflexionar y ascender a verdades autogeneradas $y$ libertarias... Las posibilidades para la estructuración de la vida cotidiana, se escogen de tal forma que se acomoden a las prioridades sensitivas y al gozo, todo ello enmarcado dentro de la circunstancialidad".

Indudablemente que en estas afirmaciones se está concediendo primacía al aprendizaje y tácitamente incorporando una relación directamente proporcional entre aprendizaje, cognición, percepción y acción, el deslinde práctico entre estos cuatro conceptos interrelacionados quedan al margen del presente trabajo. Talves, una forma de abarcar más totalidad sería estudiando la salud colectiva como una representación social por las determinaciones que ella tiene y por la forma como fue y como es construida.

En los programas educativos en salud se elude el enfoque pedagógico, los espacios físicos adecuados para acciones y los momentos para intentar educación preventiva específica; se convirtió en "costumbre normal" atiborrar o intentar embutir un montón de recomendaciones en la preconsulta o durante la espera en salas reducidas o en pasillos de las instituciones; evidentemente en máxima incomodidad y estres.

El planeamiento de las acciones educativas en salud, tanto en sus contenidos, saberes, métodos, técnicas, ayudas, objetivos, espacios, tiempos y momentos; parece legítimo hacerlas desde el conocimiento, interés, habilidades, disponibilidades, tiempos, momentos, lugares, estados emocionales, necesidades y cosmovisión del profesional de la salud, ;de hecho eso ocurre!. En la investigación MIRON et. al. $(1995)^{5}$, se encuentra una síntesis importante que retrata el sentir de las madres 
después de un contacto con los agentes e instituciones de salud.

"A mãe, ao dar entrada com seu filho nesse ambiente, é desconsiderada como portadora de saberes e prácticas no cuidado à criança. A equipe técnica assume de modo absoluto esse cuidado, investida de autoridade propiciada por sua formação profissional. À mãe cabe o lugar de coadjuvante, exigindo-se dela uma postura colaborativa com os preceitos ditados pelo pessoal hospitalar e pelas normas da instituição. $\mathrm{Na}$ aproximação da alta hospitalar a mãe é resgatada como elemento fundamental para o cuidado da criança no domicílio. Aí ocorre um esforço no sentido de oferecer informações a fim de responsabilizá-la pela completa recuperação da criança e para a manutenção de um estado saudável. Nossa experiência, como enfermeiros e docentes, permite-nos afirmar que essas informações são apresentadas em momentos de grande ansiedade, muito próximos da hora da saída do hospital e em linguagem técnica, havendo pouca ou nenhuma preocupação em verificar o entendimento dessas mães. Ao mesmo tempo, muitas vezes ocorre um descolamento entre os cuidados prescritos e as condições sociais da criança e sua família pois desconhecese sua história de vida, limitada, no sistema de saúde, aos registros biológicos/sintomatológicos. Esses saberes e práticas populares, quando relatadas pelas mães, são suficientes para o "doutor xingar", constituindo-se em mais uma violência para a mãe".

Con las ideas implicitas y explícitas, sintéticas y eclépticas que anteceden, intento con optimismo, despertar interrogantes y poner en discusión algunas causas profundas del problema y dar una interpretación al porqué esa situación ruin de la salud en América Latina, en medio del avance científico y tecnológico de la postmodernidad.

\section{HEALTH-EDUCATION: WHAT IT CAN BE BUT IT IS NOT}

Based on his knowledge and experience, the author evaluates the concept and view of the medicine, health and education practices as well as the citizen's role. Then, the author aims at inquiring and interpreting the causes of the inefficiency of the health system and its changes due to scientific and technological improvement.

KEY WORDS: evaluation, health, education

\section{REFERENCIAS BIBLIOGRÁFICAS}

01. BENSEN, S.A.T. O principio educativo no trabalho do enfermeiro. Dissertação (Mestrado) - U.F.P. 1994 e Boletín Latino Americano de Enfermería, n.15, p.4, 1995.

02. BERNSTEIN, B. Linguistic codes, hesitation phenomena and inteligence. Londres s. cd. 1971. p.43.

03. GUEVARA, C.M.A.; RODRÍGUEZ, R.J.E. Estado actual y perspectivas del programa supervivir en villavicencio. Bogotá, 1993. 248p. Tesis (Maestría) - Centro Internacional de Educación y Desarrollo Humano (CINDE) - Universidad Pedagógica Nacional (UPN).
04. MELLO, D.F.de. Opiniões de mães sobre assistência à saúde da criança de 0 a 59 meses de idade: um estudio em dois bairros de Ribeirão Preto, SP. Ribeirão Preto, 1993. p.92. Dissertação (Mestrado) - Escuela deEnfermería de Ribeirão Preto, Universidad de São Paulo.

05. MIRON, V.L.; PALHA, P.F.; RODRIGUEZ, R.J.E Saberes e prácticas das mães no cuidado à criança no pós-alta hospitalar. Ribeirão Preto, USP, 1995. 31p. (mimeo)

06. REY, G. Televisión y proceso de socialización. In:_ Televisión y violencia. Bogotá: COLCIENCIAS, 1987. p.32.

07. REY, G. El proceso de socialización. Programa de postgrado. Bogotá: CINDE, 1991. p.24.

08. VALLA, V.V.; (org.) Educação e favela: politicas para as favelas do Rio de Janeiro. Rio de Janeiro: Petrópolis: Vozes/Abrasco, 1986 p.132,134.

09. VALLA, V.V. Ibid. p. 12. 